\title{
THE MIKI-GESSEL BERNOULLI NUMBER IDENTITY
}

\author{
M. C. CRABB \\ Department of Mathematical Sciences, University of Aberdeen, Aberdeen AB24 3UE, UK
}

(Received 19 August, 2004; accepted 1 November, 2004)

\begin{abstract}
A generalization due to Gessel [3] of Miki's identity between Bernoulli numbers is shown to be a direct consequence of a functional equation for the generating function.
\end{abstract}

2000 Mathematics Subject Classification. 11B68.

1. Introduction. Recall that the Bernoulli numbers $B_{n}$ are defined by the formal power series:

$$
\mathcal{T}(X)=\frac{X}{\mathrm{e}^{X}-1}=\sum_{n \geq 0} \frac{B_{n}}{n !} X^{n} \in \mathbb{Q}[[X]] .
$$

More generally, the Bernoulli polynomials $B_{n}(\lambda) \in \mathbb{Q}[\lambda]$ are given by:

$$
\mathcal{T}_{\lambda}(X)=\frac{X \mathrm{e}^{\lambda X}}{\mathrm{e}^{X}-1}=\sum_{n \geq 0} \frac{B_{n}(\lambda)}{n !} X^{n} \in(\mathbb{Q}[\lambda])[[X]] .
$$

In [3] Gessel established the following generalization of an identity due to Miki [4].

Proposition 1.1. (Miki, Gessel et al.). For $k \geq 1$,

$$
\sum_{i+j=k ; i, j \geq 1} \frac{B_{i}(\lambda)}{i} \cdot \frac{B_{j}(\lambda)}{j}=\frac{2}{k}\left(B_{k}(\lambda) \cdot H_{k-1}+\sum_{r+2 s=k ; s \geq 1}\left(\begin{array}{l}
k \\
r
\end{array}\right) B_{r}(\lambda) \cdot \frac{B_{2 s}}{2 s}\right),
$$

where $H_{k-1}$ is the harmonic number $\sum_{l=1}^{k-1} 1 / l$.

The original result of Miki is obtained by setting $\lambda=0$; the case $\lambda=1 / 2$ reduces to an identity of Faber and Pandharipande which appeared in [2] (with a proof supplied by D. Zagier). See [3] for the details.

We shall deduce Proposition 1.1 from a functional equation for the generating function $\mathcal{T}_{\lambda}(X)$. The proof is essentially a distillation of an argument given by Dunne and Schubert in the recent paper [1].

2. Proof. Let us write

$$
\mathcal{L}(X)=\frac{X / 2}{\tanh (X / 2)}=\frac{X}{2}\left(\frac{\mathrm{e}^{X}+1}{\mathrm{e}^{X}-1}\right)=\sum_{m \geq 0} \frac{B_{2 m}}{(2 m) !} X^{2 m}=\mathcal{T}(X)+\frac{X}{2} \in \mathbb{Q}[[X]] .
$$


(The notation ' $\mathcal{\mathcal { T } _ { \lambda }}$ ' and ' $\mathcal{L}$ ' is taken from Topology: for the Todd and Hirzebruch- $L$ genera.)

It is then an elementary exercise to establish the functional equation:

$$
\mathcal{T}_{\lambda}(t X) \cdot \mathcal{T}_{\lambda}((1-t) X)=\mathcal{T}_{\lambda}(X)((1-t) \mathcal{L}(t X)+t \mathcal{L}((1-t) X)) \quad(t \in \mathbb{R}),
$$

which may be read as an identity in the $\operatorname{ring}(A[\lambda])[[X]]$, where $A$ is the ring of polynomial functions $\mathbb{R} \rightarrow \mathbb{R}$.

Now recall that

$$
\int_{0}^{1} t^{i}(1-t)^{j} \frac{d t}{t(1-t)}=\frac{(i-1) !(j-1) !}{(i+j-1) !} \text { for positive integers } i, j \geq 1,
$$

as follows, for example, from the identity $(X-Y) \int_{0}^{1} \mathrm{e}^{t X} \mathrm{e}^{(1-t) Y} d t=\mathrm{e}^{X}-\mathrm{e}^{Y}$ in $\mathbb{Q}[[X, Y]]$. It is also easy to verify that, for any integer $k \geq 1$,

$$
\int_{0}^{1}\left(-t^{k}+1-(1-t)^{k}\right) \frac{d t}{t(1-t)}=\int_{0}^{1} \frac{\left(t-t^{k}\right)+\left(1-t-(1-t)^{k}\right)}{t(1-t)} d t=2 \sum_{1 \leq l<k} \frac{1}{l}=2 H_{k-1} \text {. }
$$

By rearranging the functional equation (2.1) as

$$
\begin{aligned}
\left(\mathcal{T}_{\lambda}(t X)-1\right) \cdot\left(\mathcal{T}_{\lambda}((1-t) X)-1\right)= & \left(1-\mathcal{T}_{\lambda}(t X)+\mathcal{T}_{\lambda}(X)-\mathcal{T}_{\lambda}((1-t) X)\right) \\
& +\mathcal{T}_{\lambda}(X)((1-t)(\mathcal{L}(t X)-1)+t(\mathcal{L}((1-t) X)-1)),
\end{aligned}
$$

we obtain from the coefficient of $X^{k}$, for $k \geq 1$, the identity:

$$
\begin{aligned}
\sum_{i+j=k ; i, j \geq 1} B_{i}(\lambda) \cdot B_{j}(\lambda) \frac{t^{i}(1-t)^{j}}{i ! j !}= & \left(-t^{k}+1-(1-t)^{k}\right) \frac{B_{k}(\lambda)}{k !} \\
& +\sum_{r+2 s=k ; s \geq 1} \frac{B_{r}(\lambda)}{r !} \cdot\left((1-t) t^{2 s}+t(1-t)^{2 s}\right) \frac{B_{2 s}}{(2 s) !}
\end{aligned}
$$

in $A[\lambda]$. Multiplying this identity by $d t /(t(1-t))$ and integrating between 0 and 1 we deduce that

$\sum_{i+j=k ; i, j \geq 1} \frac{(i-1) !(j-1) !}{(k-1) ! i ! j !} B_{i}(\lambda) \cdot B_{j}(\lambda)=2 H_{k-1} \frac{B_{k}(\lambda)}{k !}+2 \sum_{r+2 s=k ; s \geq 1} \frac{(2 s-1) !}{(2 s) !(2 s) !} \cdot \frac{B_{r}(\lambda)}{r !} \cdot B_{2 s}$.

This equality is easily recast in the form (1.1).

REMARK 2.2. Further identities, of the type considered in [1] involving the gamma function, may be obtained by multiplying $(2.1)$ by $t^{x}(1-t)^{y} d t /(t(1-t))$ for any choice of real numbers $x, y \geq 0$.

\section{REFERENCES}

1. G. V. Dunne and C. Schubert, Bernoulli number identities from Quantum Field Theory, IHES preprint P/04/31, 2004 (www.ihes.fr).

2. C. Faber and R. Pandharipande, Hodge integrals and Gromov-Witten theory, Invent. Math. 139 (2000), 137-199. $75-82$.

3. I. M. Gessel, On Miki's identity for Bernoulli numbers, J. Number Theory, 110 (2005),

4. H. Miki, A relation between Bernoulli numbers, J. Number Theory 10 (1978), 297-302. 